\title{
Adjoint free four-dimensional variational data assimilation for a storm surge model of the German North Sea
}

\author{
Xiangyang Zheng ${ }^{1}$ (D) Roberto Mayerle ${ }^{1}$ - Qianguo Xing ${ }^{2}$. \\ José Manuel Fernández Jaramillo ${ }^{1}$
}

Received: 30 September 2015 / Accepted: 30 May 2016 / Published online: 15 June 2016

(c) Springer-Verlag Berlin Heidelberg 2016

\begin{abstract}
In this paper, a data assimilation scheme based on the adjoint free Four-Dimensional Variational(4DVar) method is applied to an existing storm surge model of the German North Sea. To avoid the need of an adjoint model, an ensemble-like method to explicitly represent the linear tangent equation is adopted. Results of twin experiments have shown that the method is able to recover the contaminated low dimension model parameters to their true values. The data assimilation scheme was applied to a severe storm surge event which occurred in the North Sea in December 5, 2013. By adjusting wind drag coefficient, the predictive ability of the model increased significantly. Preliminary experiments have shown that an increase in the predictive ability is attained by narrowing the data assimilation time window.
\end{abstract}

Keywords Storm surge model - Wind drag coefficient . Data assimilation · Adjoint free 4Dvar

This article is part of the Topical Collection on the 7th International Workshop on Modeling the Ocean (IWMO) in Canberra, Australia 1-5 June 2015

Responsible Editor: Yign Noh

Xiangyang Zheng

xyzheng@corelab.uni-kiel.de

1 Research and Technology Centre Westcoast, University of Kiel, Kiel, Germany

2 Yantai Institute of Coastal Zone Research, Chinese Academy of Sciences, Yantai, China

\section{Introduction}

Storm surges are exceptional water level changes caused by the strong tangent wind on the sea surface. Storm surge is one of the most destructive natural disasters in coastal areas. On February of 1962, a severe storm swept the German Bight, causing severe damage along the German North Sea coast and costing more than 300 lives in Hamburg (Von Storch et al. 2008). The hurricane Katrina hit the south coast of the USA in August 2005. The total number of fatalities directly related to the extreme event exceeded 1200 (Fritz et al. 2007). The Bay of Bengal is another coastal area susceptible to tropical storm surges. The severe storm in October 1999 killed more than 15,000 people and caused enormous property losses (Dube et al. 2009). Although much attention has been paid to coastal protection and the number of casualties has decreased considerably, storm surge remains the main natural hazard in coastal areas. Thus, accurate forecast of such events is essential for early warning. With the enhancement in computing power and improvement in the accuracy of meteorological models, systems for the nowcasting and forecasting of storm surges are at reach.

Storm surge is caused by strong meteorological forcing due to wind and low air pressure. The effect of air pressure is straightforward and easy to simulate. Proper specifications of wind shear stress is probably the most critical issue for a storm surge model but may have the most uncertainties (Bode and Hardy 1997). For most storm surge models, the wind shear stress is expressed as a quadratic function of wind speed as follows (Hydraulics 2011) :

$\tau=\rho_{a} C_{d}\left|U_{10}\right| U_{10}$ 
where $\tau$ is the wind shear stress; $\rho_{a}$ is the air density; $U_{10}$ is the wind speed $10 \mathrm{~m}$ above the sea surface; $C_{d}$ is the wind drag coefficient. $\rho_{a}$ is dependent on the air pressure, temperature, and humidity. Abdalla and Cavaleri (2002) studied the influences of $\rho_{a}$ on the wave height and found that the variations of wave height resulting from air density variability are usually smaller than $1 \mathrm{~cm}$. Therefore, air density is expected to have only minor effect on storm surge. $U_{10}$ is often obtained from meteorological models for extratropical storms (Horsburgh and Wilson 2007) or calculated by a tropical cyclone formula (Holland 1980). There has been great progress in atmospheric modeling with the development of measurements and computation ability. Although $C_{d}$ has been studied over decades, there is still no definite conclusion due to the complex mechanism of air-sea interactions. Most storm surge models (Verboom et al. 1992; Lowe et al. 2001; Weisberg and Zheng 2008) apply a linear function of $C_{d}$ with respect to $U_{10}$ proposed by many different authors (Smith and Banke 1975; Smith 1980; Wu 1980). However, these functions show major discrepancies. Lin et al. (2002) pointed out that the variability of $C_{d}$ not explained by $U_{10}$ is substantial. Weaver (2004) used the advanced circulation hurricane storm surge model (ADCIRC) to test the sensitivity of storm surges on seven different $C_{d}$. He found differences in the peak surge of up to $1.75 \mathrm{~m}$, pointing to large uncertainties.

Data assimilation has been widely used in operational weather forecast centers. By merging results from numerical models and observations, data assimilation can decrease the uncertainties of the initial conditions and improve forecast. This technique has also been used quite successfully to obtain $C_{d}$ and other model parameters for ocean models (Moore 1991; Marotzke et al. 1999; Zhang et al. 2003). Since 1950s, the application of data assimilation in weather forecast evolved from objective analysis, optimal interpolation to 3-dimensional and 4-dimensional variational method. The first three methods combine model results and observations at one given time. 4Dvar, on the other hand, extends the assimilation from a given time to a period (time window). 4Dvar is an attractive method as it takes the model into account making data assimilation consistent with model dynamic. Adjoint equations are powerful tools in 4Dvar in that it facilitates the computation of gradient of cost functions with respect to the control variables with large dimension. Yu and O'Brien (1991) used 4Dvar to estimate the $C_{d}$ values; they found that after 11 iterations $C_{d}$ converges to 0.00126 . Peng et al. (2013) used a threedimensional ocean model and its adjoint model to adjust $C_{d}$; it was found that the adjustment of $C_{d}$ can compensate for the wind data errors and improve the simulation of storm surges. Li et al. (2013) found that the optimization of initial conditions and $C_{d}$ in storm surge model was better than optimizing only one of them.
However, it is a huge undertaking to develop adjoint models for ocean models (Lawless 2012). In this paper, 4Dvar will be used to an existing storm surge model for the German Bight (Bruss et al. 2011). The proposed method is structurally similar to adjoint methods. The advantage is that it avoids the development of adjoint equations facilitating implementation. In this paper, the model and observations are described initially. This is followed by a detailed description of the adjoint free 4Dvar proposed here. Results of twin experiments carried out to validate the data assimilation method are presented. The effectiveness of the proposed method is demonstrated for a storm surge on the German North Sea coast.

\section{Model and data}

The Delft3D model suite for simulation of hydrodynamics, sediment transport, and morphodynamics in coastal waters is adopted (Hydraulics 2011). Delft3D is widely used for coastal studies (Elias et al. 2001; Mayerle and Zielke 2005; Jiao 2014). The study area is the German Bight, located in the southeast of the North Sea. Due to the coastline shape, it is one of the most susceptible areas to storm surges. In order to capture the storm surge adequately, the German Bight Model (GBM) was nested to a larger scale model covering the whole Northwest European continental shelf (CSM). The two model domains are shown in Fig. 1. The spatial resolution of the grid in the CSM is about $9 \mathrm{~km}$ and $0.5 \sim 1.7$ $\mathrm{km}$ in the GBM. The time step of the CSM and GBM is 3 and $2 \mathrm{~min}$, respectively. Similar to most storm surge models, $C_{d}$ is determined by a linear function with respect to wind speed $10 \mathrm{~m}$ above the sea surface as follows:

$C_{d}=\left(a+b U_{10}\right) 10^{-3}$

where $a=0.61, b=0.063$, which is proposed by (Smith 1980). In Eq. $2, U_{10}$ is between 6 and $22 \mathrm{~m} / \mathrm{s}$. $C_{d}$ is a constant equal to $9.88 \times 10^{-4}$ for wind speeds lower than 6.0 $\mathrm{m} / \mathrm{s} ; C_{d}$ is extrapolated for wind speeds exceeding $22 \mathrm{~m} / \mathrm{s}$.

\subsection{Bathymetry data}

The bathymetry of the North Sea is taken from the original model provided by (Verboom et al. 1992). The bathymetry near the German Bight coast has been updated by a series of measurements by the Bundesamt für Seeschifffahrt und Hydrographie (BSH), Wasser-und-Schiff fahrtsverwaltung des Bundes (WSV), Kuratorium für Forschung im Küsteningenieurwesen (KFKI), and Amt für ländliche Räume (ALR) Husum. This bathymetrical information covers the German coast for the period 1969-2009. Fernandez Jaramillo (2014). 

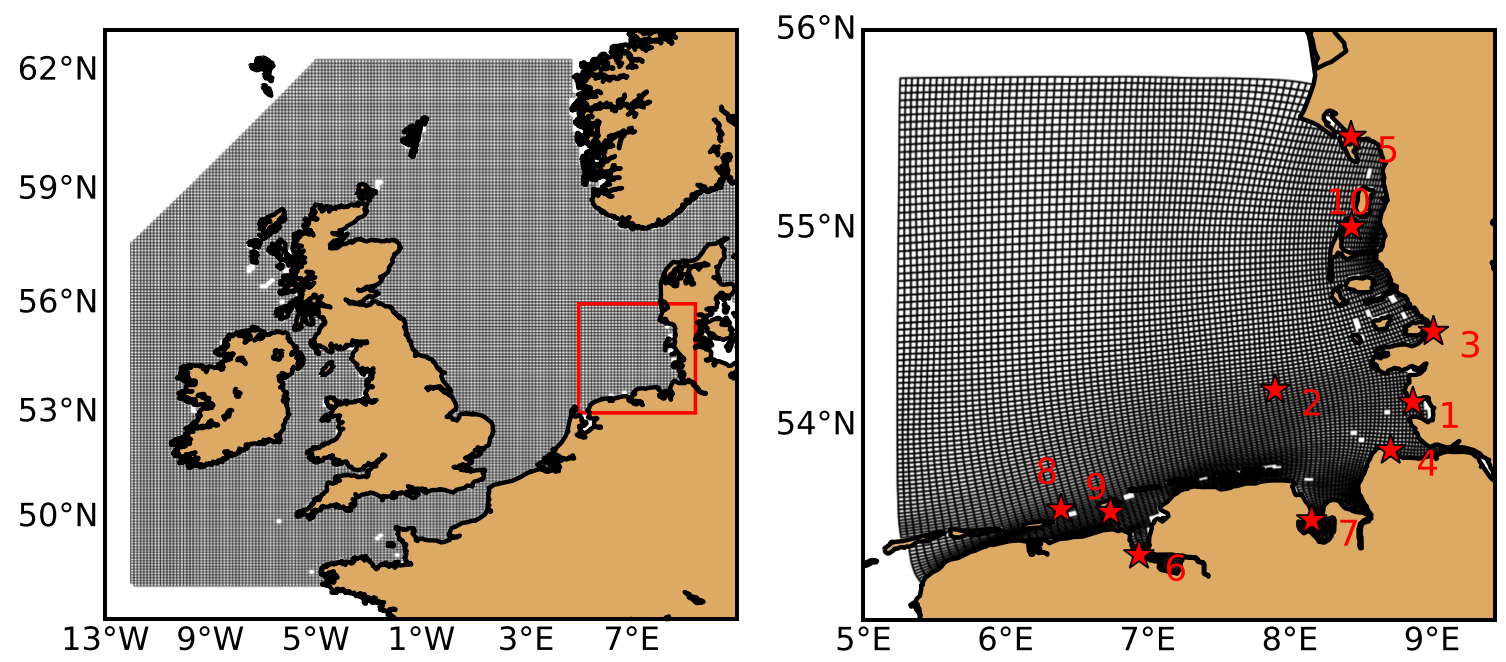

Fig. 1 Curvilinear grids of models. The left figure shows the CSM model and the right one the GBM model. Red stars mark the stations used in this paper: Buesum(1), Helgoland(2), Husum(3), Cuxhaven(4), Esbjerg(5), Delfzijl(6), Wilhelmshaven(7), Huibertgat(8), Borkum(9), Sylt(10)

\subsection{Wind and air pressure data}

In this study wind and air pressure stem from the meteorological model Consortium for Small Scale Modeling (COSMO-EU) operated by the German Weather Service center(DWD) (COSMO 2007). The spatial grid resolution is $0.0625^{\circ}$ and the time interval is $1 \mathrm{~h}$. The model was assessed on the basis of measurements at several stations along the
German North Sea coast. Figure 2 shows comparisons of modeled and observed air pressure and wind speeds at the Station Sylt in the north of Germany (Fig. 1). The root mean square deviation of wind speed is about $1.62 \mathrm{~m} / \mathrm{s}$ and the correlation coefficient is 0.90 . It was found that the meteorological model can capture the real wind field quite well thus providing adequate meteorological forcing to the storm surge model.
Fig. 2 Comparison of DWD modeled data and observed data in the early December 2013 at the station Sylt (see Figure 1)
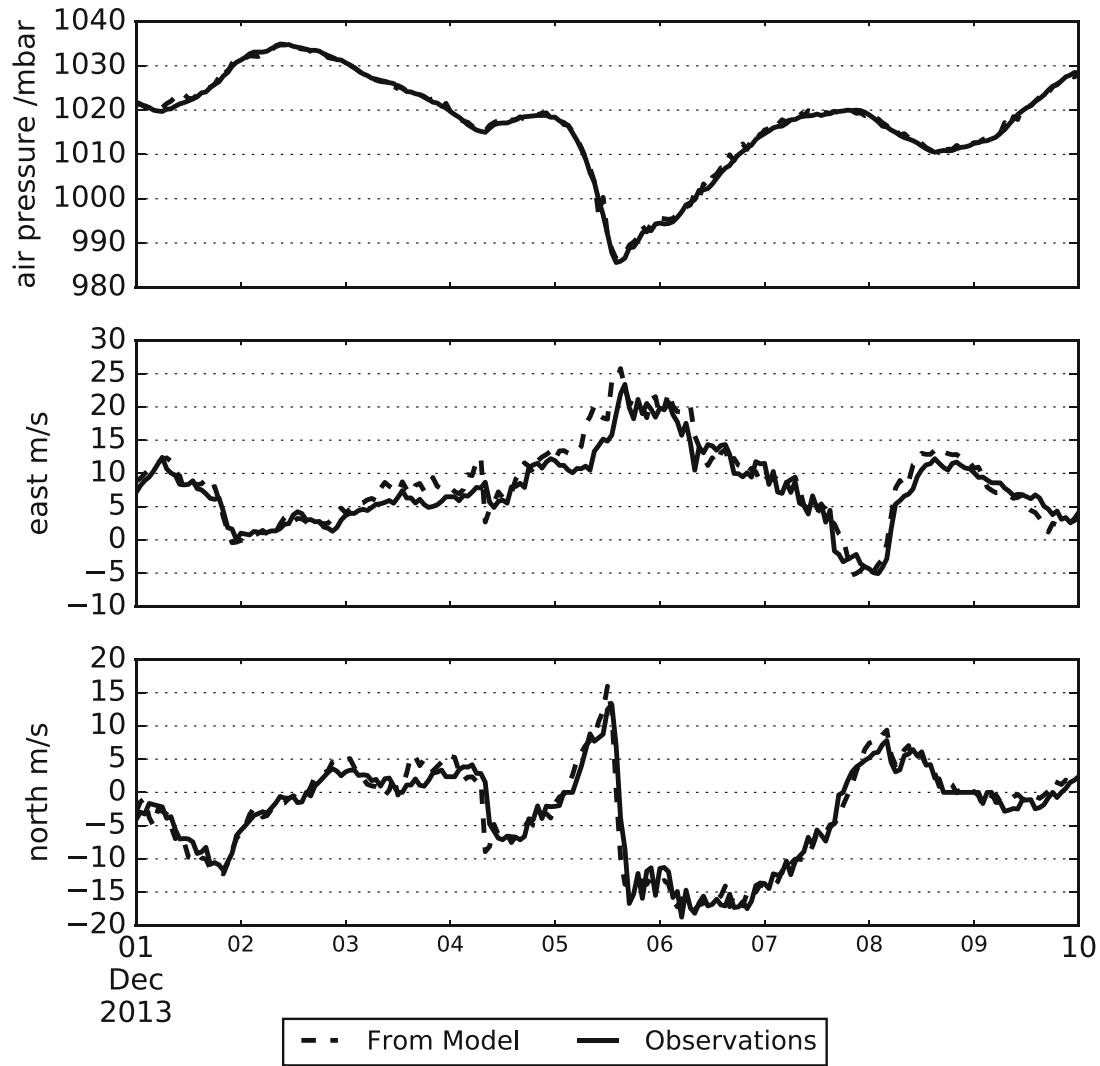


\subsection{Open boundary conditions}

Nine dominating tide harmonics from TOPEX/Poseidon (Egbert and Erofeeva 2002) were imposed at the open boundaries of the CSM . The simulated water levels from the CSM were then interpolated along the open boundaries of the GBM for forcing.

\subsection{Tidal gauges data}

In this study, water level observations with 10-min interval from several tidal gauges of the German Bight were used (see Fig. 1 right). Quality control was performed to remove the invalid data and fill out missing data.

\section{Data assimilation method}

\subsection{Dvar}

Numerical models can be denoted in a general form as follows:

$x_{i+1}=\mathcal{M}_{i}\left(x_{i}, p\right)$

where $x_{i} \in R^{n}$ is the model state vector. Storm surge models are mainly 2D, and model state includes water level and horizontal current velocity in two directions. Hence, the dimension of state $n$ is three times the number of model grid points. $i$ is the time index ranging from 0 to $N_{T} . p \in R^{k}$ is the model parameter vector, consisting of initial conditions, boundary conditions, and all other model parameters. $\mathcal{M}_{i}$ is the model forward operator. Given the model parameters $p$, the model state at $t_{i}$ is integrated to a new model state at $t_{i+1}$.

The analysis of direct observations offers another alternative to improve our understanding about the ocean. Observations of ocean state at time $t_{i}$ are denoted as $y_{i} \in R^{m}$, where $i=0,1, \cdots, N_{T}$. Normally, $m$ is much smaller than $n$ due to the difficulty of installing instruments in the ocean.

Data assimilation can combine models and observations using the statistical estimate theory. Compared with 3Dvar, 4Dvar uses observations over a certain period; model dynamics is also considered in the process of data assimilation. Therefore, results of 4Dvar are more consistent with model than 3Dvar or optimal interpolation. 4Dvar aims to find a set of model parameters which are closest to their first guess values and that generate model results closest to the observations. To this end, 4Dvar solves a minimization problem on a cost function:

$$
\begin{aligned}
J\left(p, x_{i}\right)= & \frac{1}{2}\left(p-p_{b}\right)^{T} B^{-1}\left(p-p_{b}\right)+ \\
& \frac{1}{2} \sum_{i=1}^{N_{T}}\left(H_{i} x_{i}-y_{i}\right)^{T} Q^{-1}\left(H_{i} x_{i}-y_{i}\right)
\end{aligned}
$$

where $p_{b}$ is the first guess or background values of $p ; B$ and $Q$ are weighting matrix of $p_{b}$ and $y_{i}$, respectively. They represent the uncertainties of $p_{b}$ and $y_{i}$. Therefore, terms with low uncertainties are weighted more heavily in the cost function. $N_{T}$ is the number of time steps of observations. The superscript $T$ denotes transposing matrix or vectors. $H_{i}$ is the observation operator at time $t_{i}$. In the rest part of Section 3, it is assumed that observations are much more accurate than first guess of parameters and all observations have the same accuracy. As a result, only the observation term is kept in the cost function in Eq. 4. The simplified cost function is:

$J\left(p, x_{i}\right)=\frac{1}{2} \sum_{i=1}^{N_{T}}\left(H_{i} x_{i}-y_{i}\right)^{T}\left(H_{i} x_{i}-y_{i}\right)$

$x_{i}$ between $i=1$ and $N_{T}$ are not independent but subject to the model in Eq. 3. Therefore, 4Dvar is a constraint optimization problem. It can be converted to an unconstrained optimization problem by Lagrangian multiplier methods as follows:

$$
\begin{aligned}
L\left(p, \lambda_{i}, x_{i}\right)= & \frac{1}{2} \sum_{i=1}^{N_{T}}\left(H_{i} x_{i}-y_{i}\right)^{T}\left(H_{i} x_{i}-y_{i}\right)+ \\
& \sum_{i=1}^{N_{T}} \lambda_{i-1}^{T}\left(x_{i}-\mathcal{M}_{i-1}\left(x_{i-1}, p\right)\right)
\end{aligned}
$$

where $\lambda_{i} \in R^{n}$ is the Lagrangian multiplier. The variation of Eq. 6 is

$$
\begin{aligned}
\delta L= & \sum_{i=1}^{N_{T}}\left(H_{i} x_{i}-y_{i}\right)^{T} H_{i} \delta x_{i}+ \\
& \sum_{i=1}^{N_{T}}\left(x_{i}-\mathcal{M}_{i}\left(x_{i-1}, p\right)\right)^{T} \delta \lambda_{i-1}+ \\
& \sum_{i=1}^{N_{T}}\left(\lambda_{i-1}^{T} \delta x_{i}-\lambda_{i-1}^{T} \frac{\partial \mathcal{M}_{i-1}}{\partial x_{i-1}} \delta x_{i-1}-\lambda_{i-1}^{T} \frac{\partial \mathcal{M}_{i-1}}{\partial p} \delta p\right)
\end{aligned}
$$

To get the gradient of $L$ with respect to $p$, the terms without $\delta p$ and $\delta \lambda_{i-1}$ are set to 0 , giving:

$\lambda_{i-1}-\left(\frac{\partial \mathcal{M}_{i}}{\partial x_{i}}\right)^{T} \lambda_{i}=-H_{i}^{T}\left(H_{i} x_{i}-y_{i}\right)$

Equation 8 is the so-called adjoint model. $\lambda_{i}$ is the adjoint variable. Given the first guess of model parameter $p$, the ocean model integrates forward leading to model results. $\lambda_{N_{T}}$ is then set to zero. Equation 8 is integrated backward forced by $H_{i}^{T}\left(H_{i} x_{i}-y_{i}\right)$, which is the deviations between observations and model results. All the $\lambda_{i}$ can be solved. After that $\lambda_{i}$ are substituted into equation below:

$$
\frac{\partial L}{\partial p}=\sum\left(-\frac{\partial \mathcal{M}_{i}}{\partial p}\right)^{T} \lambda_{i}
$$

Note that the gradient $\nabla_{p}^{J}=\frac{\partial L}{\partial p}$. Once $\nabla_{p}^{J}$ is calculated, gradient-based optimization methods such as the 
BFGS quasi-Newton minimization algorithm (Nocedal and Wright 2006) can be used to obtain the optimal $p$ which minimizes (5). This is an iterative process, and it stops once the termination condition is satisfied.

\subsection{Incremental 4Dvar}

4Dvar methods are extremely time-consuming and expensive computationally. They were not implemented for operational weather forecast until the incremental 4Dvar was developed. Incremental 4Dvar uses the increment of $p$, i.e., $\Delta p$ as the control variable instead of $p$. Assume that the optimal $p$ is close to the first guess of $p=p_{b}+\Delta p . \Delta x_{i}$ is the increment of $x_{i}$ due to increment of $p . \Delta x_{i+1}$ can be represented by $\Delta p$ and $\Delta x_{i}$ :

$\Delta x_{i+1}=\mathcal{M}_{i}\left(x_{i}^{b}+\Delta x_{i}, p_{b}+\Delta p\right)-\mathcal{M}_{i}\left(x_{i}^{b}, p_{b}\right)$

Apply Taylor's formula and only keep the terms of first order:

$\mathcal{M}_{i}\left(x_{i}^{b}+\Delta x_{i}, p_{b}+\Delta p\right)=\mathcal{M}_{i}\left(x_{i}^{b}, p_{b}\right)+\frac{\partial \mathcal{M}_{i}}{\partial x_{i}} \Delta x_{i}+\frac{\partial \mathcal{M}_{i}}{\partial p} \Delta p$

Substituting (11) into Eq. 10, the tangent linear equation can be obtained:

$\Delta x_{i+1}=\frac{\partial \mathcal{M}_{i}}{\partial x_{i}} \Delta x_{i}+\frac{\partial \mathcal{M}_{i}}{\partial p} \Delta p$

where $\frac{\partial \mathcal{M}_{i}}{\partial x_{i}}$ and $\frac{\partial \mathcal{M}_{i}}{\partial p}$ are linear operators. A new cost function with respect to $\Delta x_{i}$ can be obtained by substituting $x_{i}=x_{i}^{b}+\Delta x_{i}$ into Eq. 6 as follows:

$J_{1}\left(\Delta p, \Delta x_{i}\right)=\frac{1}{2} \sum_{i=1}^{N_{T}}\left(H_{i} \Delta x_{i}-d_{i}\right)^{T}\left(H_{i} \Delta x_{i}-d_{i}\right)$

$d_{i}=y_{i}-H_{i} x_{i}^{b}$. Equation 13 is minimized subject to the Eq. 12, which is a linear model. Therefore, Eq. 13 has an unique global minimum at $\Delta p^{a}$, which can be solved with BFGS. The process that solves $\Delta p^{a}$ represents a inner loop. $p$ is updated in an outer loop by $p_{1}=p^{b}+\Delta p^{a}$ and then a new inner loop starts with the new first guess $p_{1}$. The two loops proceed until $\Delta p^{a}$ is small enough.

The linear tangent (12) can be a simplified model or solved on a coarser model grid. This can reduce the computation burden and enables to apply 4Dvar in large scale models. Operational weather forecast centers such as ECMWF (Rabier et al. 2000), UK Met. office (Lorenc et al. 2000) and the ocean model ROMS (Moore et al. 2011) use incremental 4Dvar in their operational models.

\subsection{Adjoint free(AF) 4Dvar}

Adjoint methods are very powerful tools for parameter estimation. The gradients of cost functions with respect to the model parameters can be calculated by integrating both forward and adjoint models regardless of the dimension of the model parameters. Although adjoint and forward models have the same scale, the development and maintenance of adjoint models for complex ocean models are very demanding. Kalman filters are used in data assimilation for cases where the dynamics are linear. The covariance of model state evolves with model dynamics. When observations are available, the updated covariance is then used to obtain an updated model state with higher accuracy than both model and measurements. However, due to the prohibitively large dimension of the model state, they cannot be applied to ocean models. Evensen (1994) combined a Monte Carlo method with a Kalman filter method leading to the ensemble Kalman filter(ENKF). ENKF propagates the model results covariance from the spread of model results by integrating models with different initial conditions. This can replace the Kalman filter, and it is very simple to implement. Inspired by the ensemble method, we proposed a method based on the incremental 4Dvar but without an adjoint equation for parameter estimate.

In Eq. 12, $\Delta x_{i+1}$ is calculated sequentially given $\Delta x_{i}$ and $\Delta p . \Delta x_{i+1}$ can also be represented by a implicit function, as follows:

$\Delta x_{i}=M_{i}(\Delta p)$

Substituting (14) into Eq. 13, a cost function with respect to $\Delta p$ is obtained,

$J_{2}(\Delta p)=\frac{1}{2} \sum_{i=1}^{N_{T}}\left(H_{i} M_{i} \Delta p-d_{i}\right)^{T}\left(H_{i} M_{i} \Delta p-d_{i}\right)$

To calculate the gradient $\nabla_{\Delta p}^{J_{2}}$, the essential step is to represent $M_{i}$ explicitly. $M_{i}$ represents the linear tangent model, which converts perturbations of $p$ to the perturbations of model results at time $t_{i}$. The idea is to add perturbations to the parameters to be estimated just like adding perturbations to the initial condition in ENKF. Then the ensemble models are run and the perturbations of model results obtained.

Assume that the first guess of $p$ is $p_{b}$ and the corresponding model results are $x_{b}$. Then $N$ perturbations of $p$, stored in a matrix $P \in R^{k \times N}$ are generated. Adding $p_{b}$ to each column in $P$ results in $N$ models with different parameters $p$. Running these models leads to an ensemble of model results. The ensemble is denoted as $E_{i} \in R^{n \times N}$ at time $t_{i}$. Recall that $n$ is the dimension of the model state. Subtracting $x_{i}^{b}$ from each row of $E_{i}$, an ensemble perturbation of model 
state denoted as $X_{i} \in R^{n \times N}$ is obtained. Both $X_{i}$ and $P$ are small perturbations, therefore, $X_{i} \approx M_{i} P$. Since both $P$ and $X_{i}$ are available, $M_{i}$ can be estimated with the least square method. Once $M_{i}$ is estimated explicitly, the cost function (15) can be represented explicitly. It is an exact quadratic equation. Therefore, the unique optimal $\Delta p$ can be solved by BFGS. This is the inner loop in the incremental 4 Dvar. The outer loop is the same as in the incremental 4Dvar.

The difference between ENKF and the method proposed here is the way perturbations are generated. Perturbations for ENKF must follow a Gaussian distribution and the number of perturbations must be high enough for a proper representation. In the adjoint free 4Dvar, perturbations of $p$ can be any relatively small value. However, if perturbations are too small, the round-off errors become important in the $X_{i}$ and would affect the accuracy of the estimate of the linear tangent model. Therefore, it is necessary to calibrate the perturbations before implementing the data assimilation.

The model state perturbation matrix $X_{i}$ is the model generated responses to the perturbations of parameters. Thus, it is consistent with model dynamic similar to 4Dvar and Kalman filter. The proposed method is similar to Ensemble Kalman filter concerning the easy implementation. It does not need the adjoint model and is much easier than an adjoint based 4Dvar.

To avoid the under-determined problem, the number of perturbations $N$ should be larger than the dimension of model parameters $k$. For a high dimension model parameter such as initial condition, it is impossible to run $k$ models. The possible way to resolve the under-determined problem is by reducing the dimension of $p$. Singular value decomposition of $P=U S V^{T}$ is performed prior to the calculation of $M_{i}$. The first $r$ columns in $U$ are $r$ directions which account for the largest variance of $\Delta p$. A reduced parameter matrix $P_{r}$ is constructed by $P_{r}=U^{T} P, r \leq N . \Delta p_{r}$ can reserve most variance of $\Delta p$ but becomes a low dimension parameter. Optimal $\Delta p_{r}$ can be calculated by optimizing the cost function with respect to $\Delta p_{r}$. The optimal $\Delta p$ is obtained by $\Delta p=U \Delta p_{r}$.

The steps of implementation of the proposed adjoint free 4Dvar are listed :

1. Preparation of all the input files and reasonable model parameters $p^{b}$ for the model which we name here model_0.

2. Selection of the model parameters to be considered as the control variable in the data assimilation.

3. Run model_0 .

4. Generation of $\mathrm{N}$ sets of perturbations of control variables and add the perturbations to the $p^{b}$ in the model_0 and form $\mathrm{N}$ sets of models with different parameters.

5. Run the N models.
6. Estimation of the tangent linear equation by perturbations of control variables and model results and get the explicit expression of cost function.

7. Solve the cost function by BFGS and get the optimal increments for the first guess of control variables.

8. Update the control variables by adding the optimal increments to the first guess of control variables.

9. Run model_0 with updated parameters to obtain new model results.

10. Check whether the termination condition is satisfied. If true, exit from the outer loop; otherwise repeat the sequence from step 4.

In this paper, the value of cost function in Eq. 5 was used as the termination condition. If $\operatorname{abs}\left(J_{i+1}-J_{i}\right) / J_{i+1}<0.01$ ( $i$ is the index of the outer loop), the outer loop will exit.

\section{Twin experiments}

Prior to implementing the data assimilation to the storm surge model of the German Bight, several numerical twin experiments were carried out. The aim was to verify whether the method is able to improve model parameters and to check whether it is appropriate to real world cases. A typical twin experiment consists of:

Nature run: A model which represents the nature. Its parameters and model results play the role of the true values.

Pseudo observations: Sampled from the true values and added with perturbations to mimic the observations.

Control model: A model with wrong model parameters to be calibrated.

Data assimilation: A data assimilation method to improve the control model by bringing the results closer to the nature run.

Assessment of data assimilation: Assess the model results or parameters in the control model on the basis of observations and nature run.

4Dvar is a general data assimilation method and in theory, it can be used for any model parameter estimate. In this study, the CSM is adopted to the twin experiments. The observation stations are chosen arbitrarily along the coast of the German Bight. The parameters of nature run are those shown in Section 2. The aim is to improve $C_{d}$ by assimilating pseudo observations of water levels. Two types of control runs were carried out. One is obtained by perturbing only $a$ and $b$ in Equation 2 from the nature run; the other comprising of three experiments is obtained by perturbing both Chezy coefficient(uniform bottom drag coefficient) and $C_{d}$. See Table 1 for more detailed settings. In control run 1 , 
Table 1 Parameters used in nature run and control runs

\begin{tabular}{llllll}
\hline & Nature run & Control run 1 & Control run 2 & Control run 3 & Control run 4 \\
\hline ChezyU & 65 & 65 & 70 & 80 & 100 \\
ChezyV & 65 & 65 & 70 & 80 & 100 \\
$a$ & 0.61 & 0.71 & 0.71 & 0.71 & 0.71 \\
$b$ & 0.063 & 0.083 & 0.083 & 0.083 & 0.083 \\
\hline
\end{tabular}

ChezyU and ChezyV denote the Chezy coefficient $\left(\mathrm{m}^{1 / 2} \mathrm{~s}^{-1}\right)$ in the $\mathrm{U}$ and $\mathrm{V}$ direction respectively; $a$ and $b$ are the coefficients in Eq. 2

two experiments were carried out; in the first, only one station was used for data assimilation whereas the second used two stations. The aim was to test the effect of the number of observations. The observations are water level extracted from the nature run without adding perturbations. This is done as it is easier to test the ability of the data assimilation to recover $a$ and $b$ from the control runs back to the nature run.

\subsection{Control run 1}

In control run 1, model parameters that are responsible for the errors are known and data assimilation is used to improve the respective parameters. Figure 3 show the changes in the cost function obtained in the two control runs. It can be seen that both cost functions have a sharp decrease at the first and second iteration. Then they level off at zero. $a$ and $b$ are recovered to 0.61 and 0.063 exactly. This confirms the adequacy of the proposed data assimilation method for parameter estimate. It also shows that if the error sources of one model are exactly known and the observations have no error, the model parameters can be recovered to the true values even if only one observation station is used.

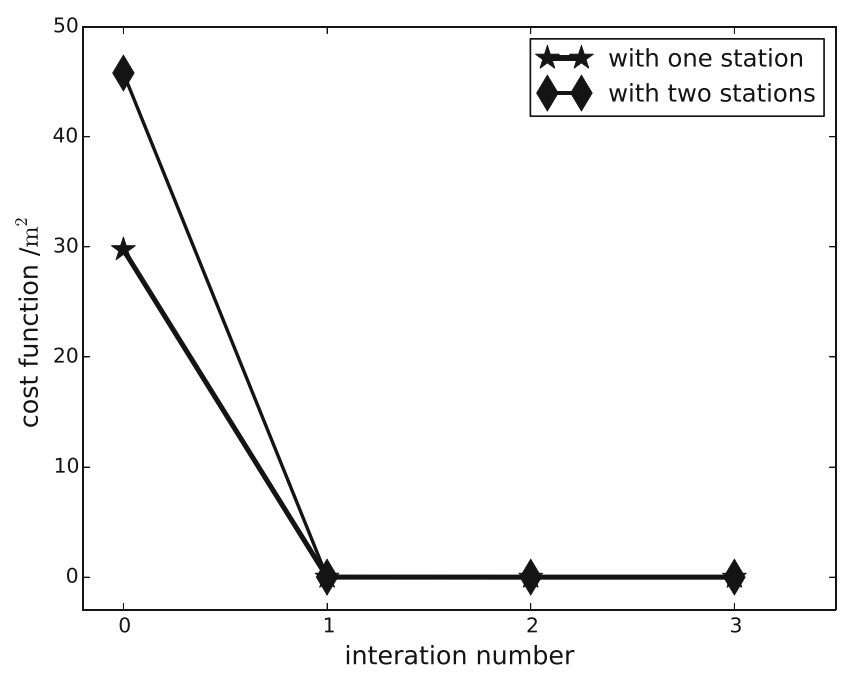

Fig. 3 Variation of cost function value in the outer loop in control run 1

\subsection{Control runs 2,3 and 4}

In control runs 2,3 , and 4 , only $C_{d}$ is adjusted in spite of the fact that there are errors in both $C_{d}$ and Chezy coefficients. Observations at two stations were used. The control runs represent more realistic situations. The difference between control runs 2, 3, and 4 is that the Chezy coefficients in control run 2 is the closest to the true value whereas in control run 4 is the deviation to the true value is largest. This led to different converged values of cost functions. They decreased to $4062,34,770$ and 168,115, respectively, (Fig. 4). The adjusted $a$ and $b$ coefficients in those control runs are shown in Fig. 5. It is found that the functions of $C_{d}$ with respect to the wind speed were improved to some extent but not recovered to the true values as in control run 1 . How much data assimilation to improve wind drag coefficient depends on the accuracy of Chezy coefficients, which were not adjusted in those control runs. The improved $C_{d}$ is closer to the true value in the control run with Chezy coefficients closer to the true value. In control run 4, the updated $a$ got even worse than its first guess, but $C_{d}$ still got closer to the true value. These experiments indicate that 4 Dvar can improve model results by adjusting some model parameters. However, it

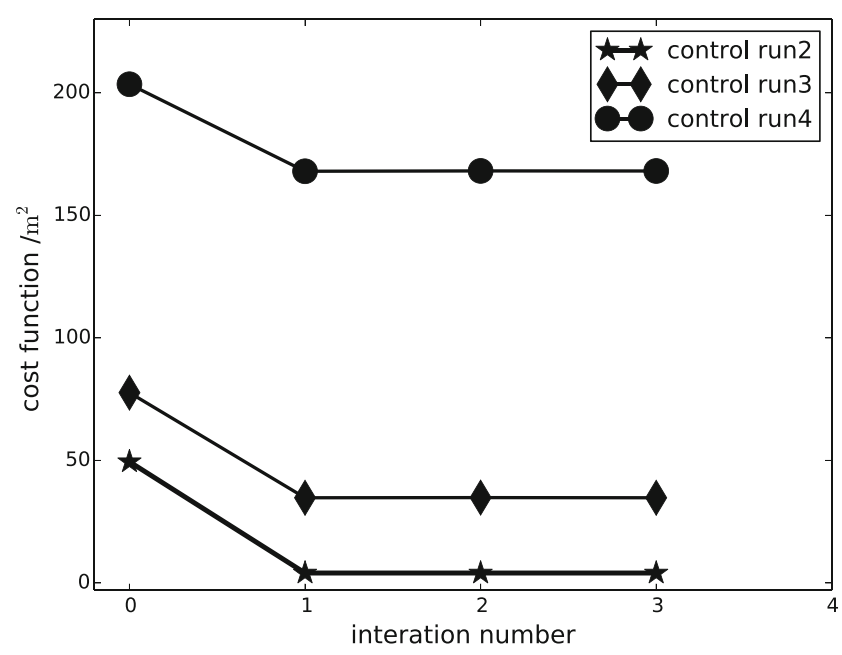

Fig. 4 Variation of cost function value in the outer loop in control runs 2,3 and 4 


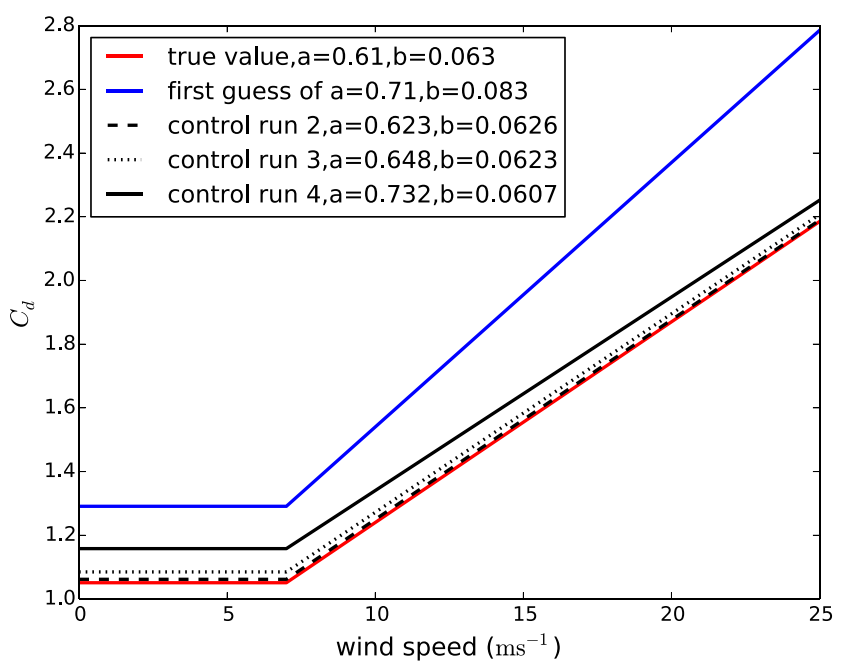

Fig. 5 Wind drag coefficient in the twin experiments

could lead to parameters that are out of the range. Therefore, it is recommended to adjust those model parameters with large uncertainties.

\section{Data assimilation on the storm surge model}

The strong storm Xaver in early December 2013 was chosen to conduct the experiments in this study. It was regarded as the largest storm in the North Sea since 1953 in terms of the wind speed. The storm cyclone started to the south of Greenland in December 4 and began to affect the North Sea on December 5, 2013.

\subsection{Tide validation}

Tide-surge interaction is very important for storm surge simulations (Horsburgh and Wilson 2007). Therefore, before modeling the storm surge, the model was validated in its ability to capture the tide. The calibrated Chezy coefficient 69.88 is used for the calculation of bottom shear stress. T_tide (Pawlowicz et al. 2002) was used for tidal harmonic analysis of modeled and observed water level. Figure 6 shows the comparisons of the amplitudes and phases of the dominating harmonics from model results and tidal gauges. M2 is the largest tidal component at all the five tidal gauges, and the model can capture the observations very well. S2 and $\mathrm{O} 1$ also have good agreement between model and observations.

\subsection{Adjustment of wind drag coefficient}

\subsubsection{Data assimilation setting}

Storm surge is mainly caused by strong wind shear stress on the sea surface. The estimate of wind shear stress is essential to skill of storm surge models. As mentioned in Section 1, wind drag coefficient $C_{d}$ is still poorly understood and
Fig. 6 Comparison of measured and modeled amplitude and phase at stations Buesum, Helgoland(Helgo), Husum, Wittduen(Witt), Bremerhaven(Brem)
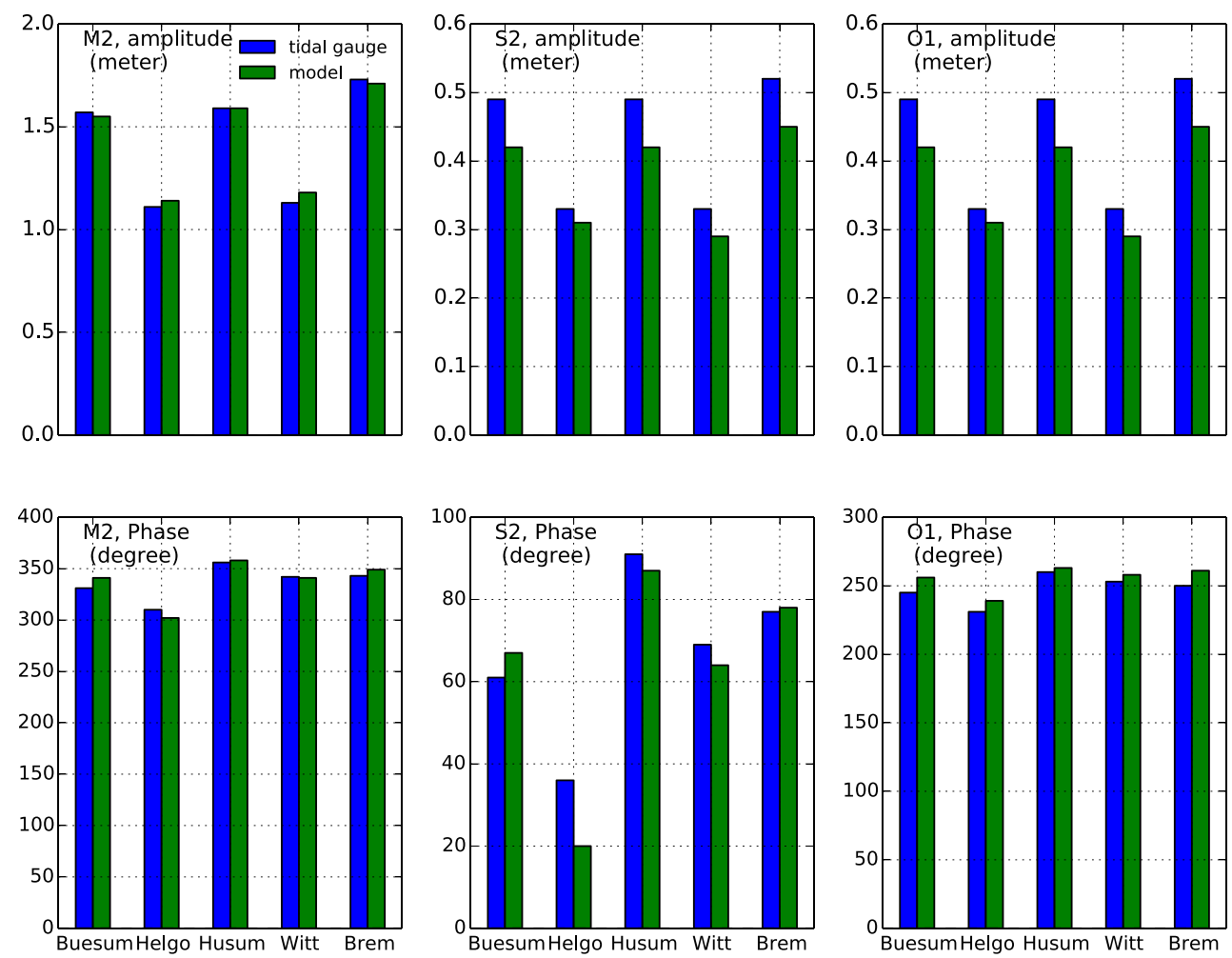
could affect storm surge model skill significantly. The initial conditions is less important than wind shear stress and open boundary in a 2D storm surge model. Sensitivity tests show that models with cold start give almost the same results if the initial simulation time is two days before the surge period. Therefore, the parameter to be improved by data assimilation is $C_{d}$. There are two control variables, i.e., $a$ and $b$ in Eq. 2. Normally, the accuracy of tidal gauges is very high. According to Woodworth and Smith (2003), the accuracy of a new radar tidal gauge and a conventional bubbler pressure gauge are comparable and in the order of $0.01 \mathrm{~m}$. The water level data used in this paper were resampled every $10 \mathrm{~min}$. This may decrease the accuracy of the data. Therefore, an accuracy of $0.03 \mathrm{~m}$ was specified for all the observations used for data assimilation. It is more difficult to specify the accuracy of $a$ and $b$. There is a brief review on the derivation of the linear relation between $C_{d}$ and $U_{10}$ in the Appendix. For more details, see Guan and Xie (2004).

Wind drag coefficient $C_{d}$ is not only related to wind speed, but also closely related with sea state. In a storm, the sea state becomes much more complex than under a normal weather condition. According to the derivation in the Appendix, more uncertainties are brought to the value of $b$ due to the uncertainties of Charnock coefficient $\alpha . a$ is independent of wave state according to the Eq. A.6. Smith (1980) mentioned that the increase in his formula is statistically significant and well above $99.9 \%$, indicating that $a$ has a higher accuracy. Therefore, 0.03 and 0.01 were adopted for the accuracies of $a$ and $b$, respectively.

The water level data from the tidal gauges Helgoland and Buesum were used for data assimilation purposes. The tidal gauge Cuxhaven, Husum, Huibertgat, and Delfzijl were used for validation of data assimilation. The time window of data assimilation covers the period of the storm surge in the German Bight from 2013-12-05 12:00 UTC to 2013-12-06 12:00 UTC.

Initial conditions are not in the control variables. In order to properly estimate $M_{i}$ in Eq. 14 for $a$ and $b$, the storm surge model should run before the time window. The period between the starting time of storm surge model and starting time of time window is a warm-up period for $M_{i}$. Sensitivity tests show that $M_{i}$ can converge if warm-up period is long enough. In this paper, the storm surge model starts 4 days before 2013-12-05 12:00 UTC with cold start. Figure 7 shows a flow chart of the data assimilation scheme implemented in the storm surge model.

\subsubsection{Results and discussions}

Figure 8 shows the variation of the cost function. It can be seen that in the data assimilation time windows the modeled water level got closer to the observations at the stations Helgoland and Buesum. The time series of the water level

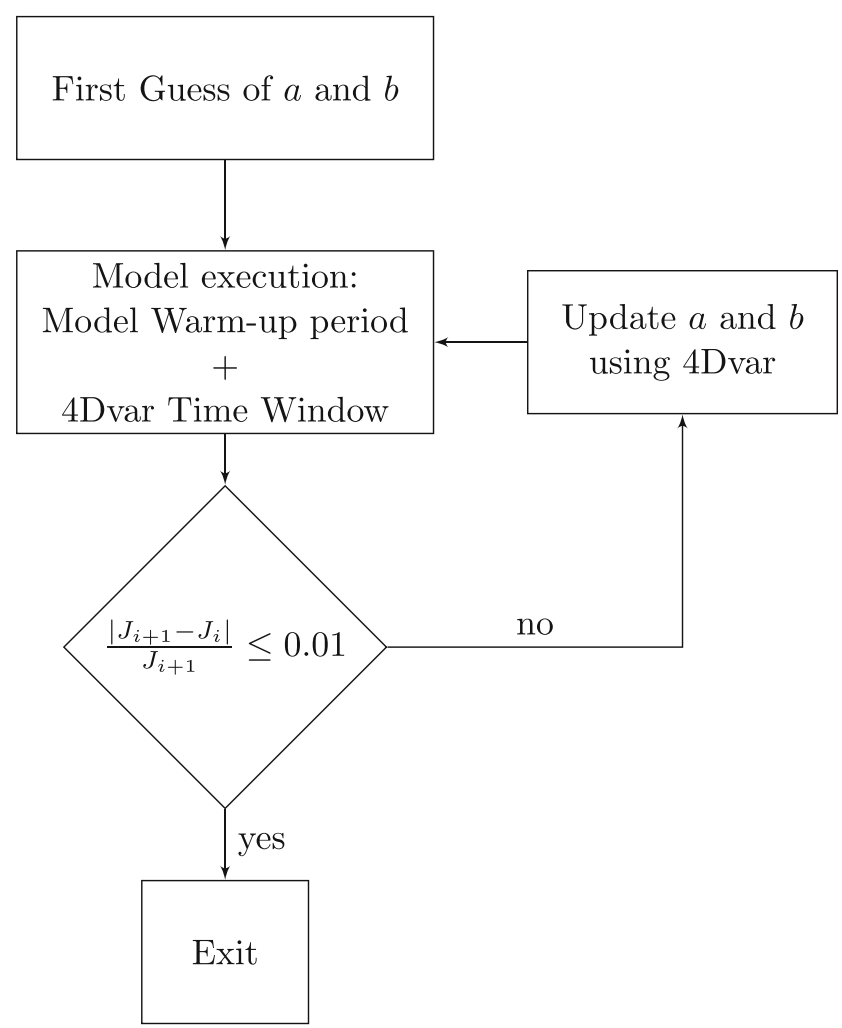

Fig. 7 Adopted data assimilation scheme

at the six stations are shown in Fig. 9. Compared with tidal gauges measured data, the storm surge model with Smith formula underestimates the water level at all the six stations. Data assimilation made the model results closer to the observations. Table 2 shows the root mean square deviations (RMSD) between modeled and observed water level. The relative RMSD reductions at the six stations range from

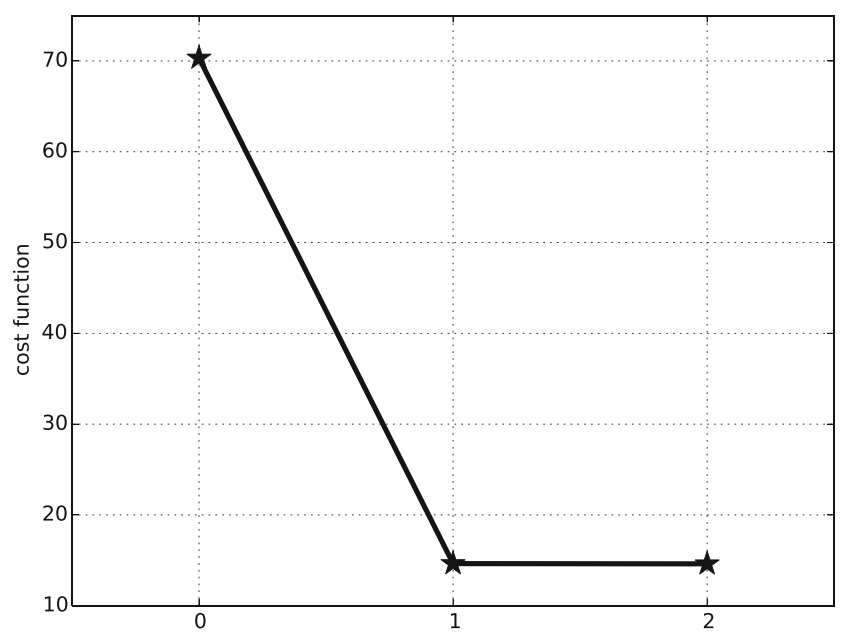

Fig. 8 Cost function of data assimilation with time window from 2013-12-05 12:00 UTC to 2013-12-06 12:00 UTC 

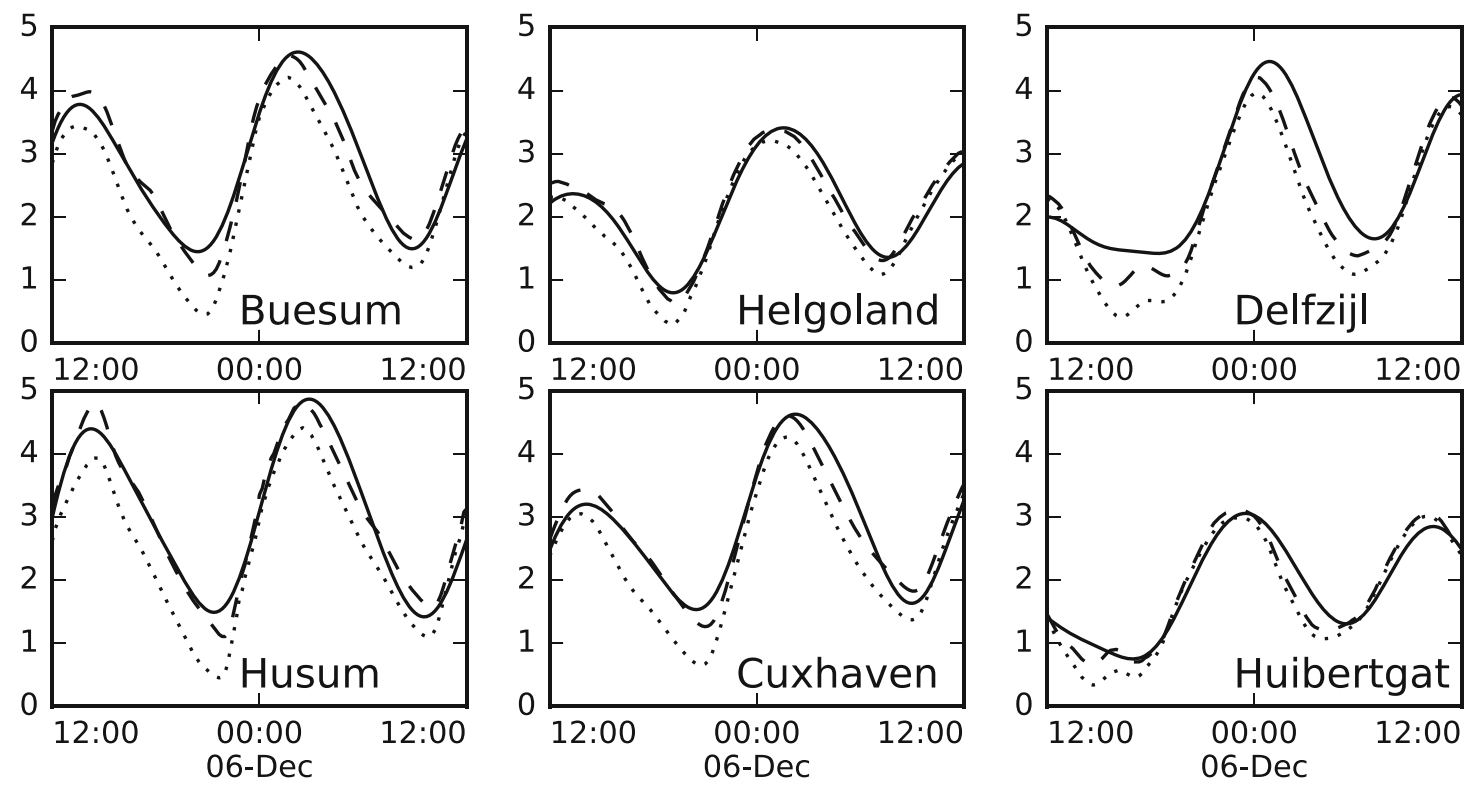

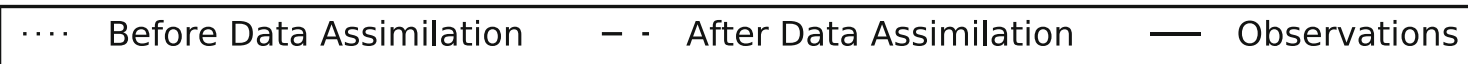

Fig. 9 Time series of water level $(\mathrm{m})$ in the storm. The solid line represents the tidal gauge measured water level; the dotted line the model results with first guess of $C_{d}$; the dashed line the model results with the improved $C_{d}$

30.6 to $63.1 \%$ in the period of time window. More improvements occurred at the stations Buesum, Helgoland, Husum and Cuxhaven between 2013-12-05 20:00 UTC and 201312-06 06:00 UTC. However, the peak is overestimated at the four stations at about 2013-12-05 14:00 UTC. This means the updated $C_{d}$ is too high for this period. The wind coefficient over the sea surface has a very complicated relation with wind, sea state, and atmospheric structures. $C_{d}$ is simply parameterized as a linear function of wind speed and it is uniform spatially and constant in time. This parameterization scheme can work well under normal weather conditions. However, in a stormy weather, the high wave makes the sea surface very rough and the sea state changes more rapidly. Hence, the 24-h time window used here may be too long for the data assimilation of $C_{d}$.

It assumes that within a short period the sea state does not change much and that it can be represented by the linear function of wind speed. Another four data assimilation were carried out with time windows of $6 \mathrm{~h}$. The time windows are 0512-0518, 0518-0600, 0600-0606, and 0606-0612, respectively. The first two digits represent the day and the last two the hour. For convenience, we named the model with first guess of $C_{d}$ as model I, the model with 24-h data assimilation time window as model II and the model with 6-h time window model III. Figure 10 shows the time series of water level at stations Buesum and Husum. Compared to model I, both model II and III improved the simulations significantly. Notably model III relieved the overestimated water level peak at about 2013-12-05 14:00 UTC. Besides better results were obtained between 2013-12-06 02:00 UTC and 2013-12-06 06:00 UTC. The root mean square deviations of water level at the six stations in the three models are listed in Table 3. At Buesum and Helgoland, which are the stations used for data assimilation, the 6-h time window improves the predictions quite clearly in the time windows 0512-0518 and 0600-0606; in another two
Table 2 Root mean square deviation of water level $(\mathrm{m})$ between 2013-12-05 12:00 UTC and 2013-12-06 12:00 UTC before and after data assimilation

\begin{tabular}{llll}
\hline & Before DA & After DA & Relative change \\
\hline Buesum & 0.619 & 0.264 & $57.3 \%$ \\
Helgoland & 0.319 & 0.176 & $45.0 \%$ \\
Delfzijl & 0.708 & 0.447 & $36.9 \%$ \\
Cuxhaven & 0.613 & 0.249 & $59.4 \%$ \\
Huibertgat & 0.325 & 0.225 & $30.6 \%$ \\
Husum & 0.650 & 0.240 & $63.1 \%$
\end{tabular}


Table 3 Root mean square deviations (RMSD) between measured and modeled water levels

\begin{tabular}{llllllll}
\hline Time window & Model & Buesum & Helgoland & Delfzijl & Husum & Cuxhaven & Huibertgat \\
\hline $0512-0518$ & I & 0.514 & 0.337 & 0.721 & 0.661 & 0.516 & 0.399 \\
& II & 0.198 & 0.162 & 0.373 & 0.177 & 0.168 & 0.172 \\
& III & 0.101 & 0.098 & 0.462 & 0.162 & 0.139 & 0.223 \\
$0518-0600$ & I & 0.757 & 0.290 & 0.491 & 0.818 & 0.703 & 0.127 \\
& II & 0.272 & 0.138 & 0.226 & 0.210 & 0.193 & 0.171 \\
$0600-0606$ & III & 0.240 & 0.141 & 0.182 & 0.162 & 0.162 & 0.186 \\
& I & 0.724 & 0.401 & 1.068 & 0.665 & 0.759 & 0.469 \\
& II & 0.337 & 0.166 & 0.747 & 0.275 & 0.346 & 0.321 \\
$0606-0612$ & III & 0.209 & 0.090 & 0.558 & 0.225 & 0.200 & 0.238 \\
& I & 0.416 & 0.228 & 0.315 & 0.378 & 0.419 & 0.135 \\
& II & 0.233 & 0.222 & 0.203 & 0.279 & 0.249 & 0.194 \\
& III & 0.218 & 0.209 & 0.213 & 0.226 & 0.226 & 0.178 \\
\hline
\end{tabular}

time windows, the improvements were only minor. At station Husum and Cuxhaven, improvements happened in all the four time windows using 6-h time window and the relative changes from 24- to 6-h time window ranged between 8 and $42.2 \%$. However, in the station Delfzijl and Huibertgat, which are far from the stations used for data assimilation, obvious improvements are only in the time window 06000606; this may be due to the spatial variability of wind drag coefficient.

The adjusted values of $a$ and $b$ in the different time windows are shown in the Table 4. The $a$ values changed around its first guess 0.61 in Smith formula. However, the updated $b$ values are much larger than in the Smith formula. They can give larger wind stress to the model during a strong wind and improve the storm surge simulation. In model III, $a$ and $b$ were different in the various time windows, indicating the temporal variability of the wind drag coefficient. Johnson and Vested (1992) proposed a hybrid wind drag coefficient model by taking wave state into account. They performed wave simulations for a constant winds of $24 \mathrm{~m} / \mathrm{s}$ from west over the North Sea. Based on the simulated wave, a distribution of $C_{d}$ is resulted over the North Sea. Wind drag coefficients ranging from 0.0026 to 0.0032 resulted. Figure 2 shows that in the period between 2013-12-05 12:00 UTC and 2013-12-06 12:00 UTC the wind speeds on the German Bight was around 22 to $25 \mathrm{~m} / \mathrm{s}$. Our results are comparable to Johnson and Vested (1992). For wind speed of $24 \mathrm{~m} / \mathrm{s}, C_{d}$ calculated from Smith formula is 0.0021 and equal to 0.0033 updated from model II. It is found that Smith formula underestimates $C_{d}$ and data assimilation can update $C_{d}$ in a more reasonable way.

Li et al. (2013) used 4Dvar to improve $C_{d}$ in the South China Sea in the period of typhoon Hagupit; they also got a set of different updated values for $a$ and $b$ for different time windows. The largest one is equal to 0.946 and 0.145 . Peng et al. (2013) also studied the wind drag coefficient in typhoon Hagupit with 4Dvar; the updated $a$ and $b$ values also varied significantly depending on the data assimilation time window, model resolution, and error sources. As mentioned above, the transfer of momentum and energy from
Fig. 10 Time series of water level $(\mathrm{m})$ in the storm at Buesum and Husum. The red line is the observations, The solid black line the model results with first guess of $C_{d}$; the dotted black line the model results with $C_{d}$ updated with 6-hour time windows; the dashed black line the model results with $C_{d}$ updated with 24-hour time windows
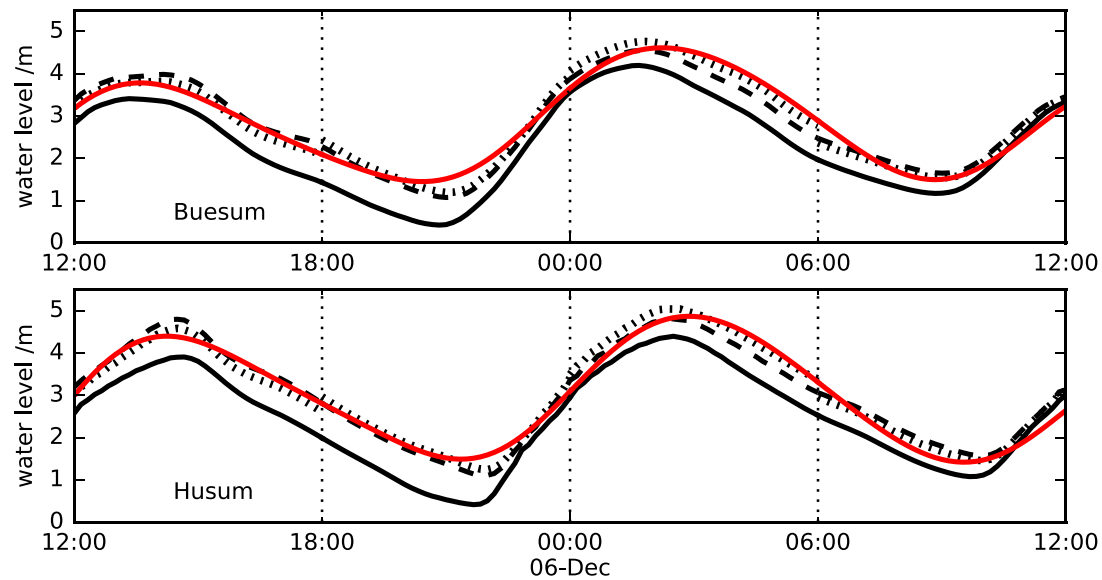

Model I - - Model II $\quad \because$ י 
Table 4 Updated $a$ and $b$ coefficients for within the different time windows

\begin{tabular}{lllll}
\hline Time window & $\begin{array}{l}\text { Model II } \\
\mathrm{a}\end{array}$ & $\mathrm{b}$ & $\begin{array}{l}\text { Model III } \\
\mathrm{a}\end{array}$ & $\mathrm{b}$ \\
\hline $0512-0518$ & 0.6244 & 0.1131 & 0.6066 & 0.1006 \\
$0518-0600$ & 0.6244 & 0.1131 & 0.6475 & 0.1222 \\
$0600-0606$ & 0.6244 & 0.1131 & 0.5726 & 0.1473 \\
$0606-0612$ & 0.6244 & 0.1131 & 0.5497 & 0.1051 \\
\hline
\end{tabular}

air to the ocean is a very complicated process. The linear relation between the $C_{d}$ and the wind speed is an approximation. In a super strong storm, the linear function of $C_{d}$ may become invalid; then $a$ and $b$ will lose their physical meanings and become just ordinary parameters of the storm surge models. Liu and Lv (2011) studied the spatially varying drag coefficients in a typhoon storm and found that the relation between the wind drag coefficient and wind speed is not clear. A more meaningful way is to use a flow-wave-air coupled model with proper wind drag coefficient parameterization schemes for storm surge simulations. Bruss and Mayerle (2009) found that it was much better to use $C_{d}$ from a wave model than from the linear function of $C_{d}$ with respect to wind speed. The data assimilation cannot repair the flaws in the storm surge model, but it can improve the model results by adjusting $a$ and $b$. The smaller improvements at the station Delfzijl and Huibertgat indicated that the improvements are limited to the surrounding areas of the data assimilation stations due to the local effects of $C_{d}$. Therefore, more stations are necessary for a large study area.

\section{Conclusion}

Combining the ideas of ensemble Kalman filter and incremental 4Dvar, a data assimilation method was developed for the parameter estimate in the storm surge models. An iterative processes to find the global minimum solution for a cost function is proposed. The method is similar to incremental 4Dvar but uses a set of perturbations to estimate the tangent linear equation and therefore represents the tangent linear equation and adjoint equation explicitly. This can avoid the development of the adjoint model, which is highly difficult and time-consuming for an ocean model. The twin experiments proved the effectiveness of the data assimilation method. A more realistic twin experiment revealed that the method can also improve model results even if the error sources in a given model are not well known. In order to avoid the unreasonable adjustment, it is better to choose model parameters with more uncertainty as the control variables. The adjoint free 4Dvar is very easy to implement on any ocean models. The models with perturbed parameters can run in parallel independently. Therefore, the computation time is comparable to an adjoint based 4 Dvar.
In a storm surge model, $C_{d}$ is widely recognized as the most uncertain model parameter. The Smith formula for $C_{d}$ works well under normal weather conditions, but leads to underestimates of water levels in a strong storm as shown in this paper. This is most probably caused by the invalidity of Smith formula in an extremely storm due to the shortage of wind stress measurements during strong storm. We used the AF 4Dvar to adjust the linear relation between the wind speed and $C_{d}$. The updated wind drag can improve the simulation very effectively. The data assimilation time window is important for the wind drag data assimilation due to the large temporal variability in a storm. A short time window may improve the estimate of $C_{d}$. In the future, data assimilation will be used to test $C_{d}$ for more storms to improve the relation between $C_{d}$ and wind speed. The proposed adjoint free method will also be used to high dimension parameters, such as initial conditions of a 3D baroclinic ocean model, in which errors of initial conditions play much greater roles than in a storm surge model.

Acknowledgments This study has been conducted using the tidal gauges data of Copernicus Marine Service Products

\section{Appendix}

The wind profile follows approximately a logarithm curve with height in the atmospheric boundary layer which can be expressed as:

$U(z)=\frac{u_{*}}{\kappa} \ln \left(\frac{z}{z_{0}}\right)$

where $U(z)$ is the wind speed at the height of $z ; z_{0}$ is the aerodynamic surface roughness; $\kappa=0.4$ is the von Karman constant; $u_{*}$ is the wind friction velocity defined by:

$u_{*}=\sqrt{\frac{\tau}{\rho_{a}}}$

Combining Eqs. 1 and A.2, a relation between $u_{*}$ and $C_{d}$ can be obtained as follows:

$u_{*}^{2}=C_{d} U_{10}^{2}$

Combining (A.1) and Eq. A.3 yields the relation between $z_{0}$ and $C_{d}$,

$z_{0}=z_{10} \exp \left(-\kappa / C_{d}^{1 / 2}\right)$ 
where $z_{10}=10 \mathrm{~m}$. Charnock (1955) proposed a relation between $z_{0}$ and $u_{*}$, i.e., $g z_{0} / u_{*}^{2}=\alpha$, where $g$ is the gravitational acceleration and $\alpha$ is the Charnock coefficient. Charnock (1955) took it as a constant. Combining all the above equations, a relation between $C_{d}$ and $U_{10}$ is obtained as below,

$\alpha^{1 / 2} U_{10} /\left(g z_{10}\right)^{1 / 2}=C_{d}^{-1 / 2} \exp \left(-\frac{\kappa}{2} C_{d}^{-1 / 2}\right)$

Equation A.5 is almost a linear function for $C_{d}$ values in the range of $1.0 \times 10^{-3}$ to $4.0 \times 10^{-3}$,

$C_{d}=\left(a+b U_{10}\right)$

where $b=0.475 \alpha^{1 / 2}$. Several values of $\alpha$ have been proposed; $\alpha=0.012$ (Charnock 1955); $\alpha=0.0144$ (Garratt 1977) $; \alpha=0.035$ (Kitaigorodskii and Volkov 1965). Stewart (1974) suggested a dependency of $\alpha$ with the wave state. Hsu (1974) related $\alpha$ to the wave steepness and Donelan et al. (1993) to the wave age.

\section{References}

Abdalla S, Cavaleri L (2002) Effect of wind variability and variable air density on wave modeling. J Geophys Res: Oceans (1978-2012) 107(C7):17-1

Bode L, Hardy TA (1997) Progress and recent developments in storm surge modeling. J Hydraul Eng 123(4):315-331

Bruss G, Gönnert B, Mayerle R (2011) Extreme scenarios at the german north sea coast a numerical model study. Coast Eng Proc 1(32):26

Bruss G, Mayerle R (2009) Investigations on the influence of the wind drag coefficient in storm surge models. In: Proceedings of the 3rd International Conference in Ocean Engineering, pp 325-332

Charnock H (1955) Wind stress on a water surface. Q J R Meteorol Soc 81(350):639-640

COSMO (2007) Operations at dwd. Technical report, cosmo-eu

Donelan MA, Dobson FW, Smith SD, Anderson RJ (1993) On the dependence of sea surface roughness on wave development. J Phys Oceanogr 23(9):2143-2149

Dube S, Jain I, Rao A, Murty T (2009) Storm surge modelling for the bay of bengal and arabian sea. Nat Hazards 51(1):3-27

Egbert GD, Erofeeva SY (2002) Efficient inverse modeling of barotropic ocean tides. J Atmos Ocean Technol 19(2):183-204

Elias E, Walstra D, Roelvink J, Stive M, Klein M (2001) Hydrodynamic validation of delft $3 \mathrm{~d}$ with field measurements at egmond. In: Coastal Engineering Conference, vol 3. Asce American Society Of Civil Engineers, pp 2714-2727

Evensen G (1994) Sequential data assimilation with a nonlinear quasigeostrophic model using monte carlo methods to forecast error statistics. J Geophys Res: Oceans 99(C5):10143-10162

Fernandez Jaramillo JM (2014). In: Development of an Operational System for a Coastal Area on the German North Sea using Artificial Intelligence. PhD Thesis, Kiel, Christian-AlbrechtsUniversität, Dissertation

Fritz HM, Blount C, Sokoloski R, Singleton J, Fuggle A, McAdoo BG, Moore A, Grass C, Tate B (2007) Hurricane katrina storm surge distribution and field observations on the mississippi barrier islands. Estuar Coast Shelf Sci 74(1):12-20

Garratt J (1977) Review of drag coefficients over oceans and continents. Monthly Weather Rev 105(7):915-929
Guan C, Xie L (2004) On the linear parameterization of drag coefficient over sea surface. J Phys Oceanogr 34(12):2847-2851

Holland GJ (1980) An analytic model of the wind and pressure profiles in hurricanes. Monthly Weather Rev 108(8):1212-1218

Horsburgh K, Wilson C (2007) Tide-surge interaction and its role in the distribution of surge residuals in the north sea. J Geophys Res: Oceans (1978-2012) 112(C8)

Hsu S-A (1974) A dynamic roughness equation and its application to wind stress determination at the air-sea interface. J Phys Oceanogr 4(1): $116-120$

Hydraulics D (2011) User manual delft3d-flow: Wl. Delft Hydraulics

Jiao J (2014) Morphodynamics of Ameland Inlet: Mediumterm Delft3D Modelling

Johnson H, Vested H (1992) Effects of water waves on wind shear stress for current modeling. J Atmos Ocean Technol 9(6):850 861

Kitaigorodskii S, Volkov YA (1965) On the roughness parameter of the sea surface and the calculation of momentum flux in the nearwater layer of the atmosphere. Izv Atmos Oceanic Phys 1:973-988

Lawless AS (2012) Variational data assimilation for very large environmental problems. Large Scale Inverse Problems: Computational Methods and Applications in the Earth Sciences, Radon Series on Computational and Applied Mathematics 13:55-90

Li Y, Peng S, Yan J, Xie L (2013) On improving storm surge forecasting using an adjoint optimal technique. Ocean Model 72:185197

Lin W, Sanford LP, Suttles SE, Valigura R (2002) Drag coefficients with fetch-limited wind waves*. J Phys Oceanogr 32(11):30583074

Liu M, Lv X (2011) Study on the drag coefficient in the simulation of storm surges with adjoint method. Oceanol Limnol Sin 1:002

Lorenc A, Ballard S, Bell R, Ingleby N, Andrews P, Barker D, Bray J, Clayton A, Dalby T, Li D et al (2000) The met. office global threedimensional variational data assimilation scheme. Q J R Meteorol Soc 126(570):2991-3012

Lowe JA, Gregory JM, Flather RA (2001) Changes in the occurrence of storm surges around the United Kingdom under a future climate scenario using a dynamic storm surge model driven by the hadley centre climate models. Clim Dyn 18(3-4):179-188

Marotzke J, Giering R, Zhang KQ, Stammer D, Hill C, Lee T (1999) Construction of the adjoint mit ocean general circulation model and application to atlantic heat transport sensitivity. J Geophys Res: Oceans 104(C12):29529-29547

Mayerle R, Zielke W (2005) Promorph-predictions of medium-scale morphodyanimcs: project overview and executive summary. Die Küste 69:1-24

Moore AM (1991) Data assimilation in a quasi-geostrophic openocean model of the gulf stream region using the adjoint method. J Phys Oceanogr 21(3):398-427

Moore AM, Arango HG, Broquet G, Powell BS, Weaver AT, ZavalaGaray J (2011) The regional ocean modeling system (roms) 4-dimensional variational data assimilation systems: Part i-system overview and formulation. Prog Oceanogr 91(1):34-49

Nocedal J, Wright S (2006) Numerical optimization. Springer Science $\&$ Business Media

Pawlowicz R, Beardsley B, Lentz S (2002) Classical tidal harmonic analysis including error estimates in matlab using t_tide. Comput Geosci 28(8):929-937

Peng S, Li Y, Xie L (2013) Adjusting the wind stress drag coefficient in storm surge forecasting using an adjoint technique. J Atmos Ocean Technol 30(3):590-608

Rabier F, Järvinen H, Klinker E, Mahfouf J-F, Simmons A (2000) The ecmwf operational implementation of four-dimensional variational assimilation. i: experimental results with simplified physics. Q J R Meteorol Soc 126(564):1143-1170 
Smith S, Banke E (1975) Variation of the sea surface drag coefficient with wind speed. Q J R Meteorol Soc 101(429):665-673

Smith SD (1980) Wind stress and heat flux over the ocean in gale force winds. J Phys Oceanogr 10(5):709-726

Stewart R (1974) The air-sea momentum exchange. Bound-Layer Meteorol 6(1-2):151-167

Verboom G, de Ronde J, van Dijk R (1992) A fine grid tidal flow and storm surge model of the north sea. Cont Shelf Res 12(2-3):213 233

Von Storch H, Gönnert G, Meine M (2008) Storm surge-an option for hamburg, Germany, to mitigate expected future aggravation of risk. Environ Sci Policy 11(8):735-742

Weaver RJ (2004) Effect of wave forcing on storm surge. Master's Thesis, University of Florida
Weisberg RH, Zheng L (2008) Hurricane storm surge simulations comparing three-dimensional with two-dimensional formulations based on an ivan-like storm over the tampa bay, florida region. J Geophys Res: Oceans (1978-2012) 113(C12)

Woodworth PL, Smith DE (2003) A one year comparison of radar and bubbler tide gauges at liverpool. Inter Hydrogr Rev 4(3)

Wu J (1980) Wind-stress coefficients over sea surface near neutral conditions - a revisit. J Phys Oceanogr 10(5):727-740

Yu L, O'Brien JJ (1991) Variational estimation of the wind stress drag coefficient and the oceanic eddy viscosity profile. J Phys Oceanogr 21:709-719

Zhang A, Wei E, Parker BB (2003) Optimal estimation of tidal open boundary conditions using predicted tides and adjoint data assimilation technique. Cont Shelf Res 23(11-13):1055-1070 